\title{
Special Section Guest Editorial: Nanostructured thin films: Fabrication, characterization, and application
}

\author{
Raúl J. Martín-Palma ${ }^{a}$ and Yi-Jun Jen ${ }^{b}$ \\ ${ }^{a}$ Universidad Autónoma de Madrid, Departamento de Física Aplicada, Campus de \\ Cantoblanco, 28049 Madrid, Spain, and The Pennsylvania State University, Department of \\ Materials Science and Engineering, University Park, Pennsylvania 16802 \\ rauljose.martin@uam.es \\ ${ }^{b}$ National Taipei University of Technology, Department of Electro-Optical Engineering, \\ No. 1, Sec. 3, Chung-Hsiao E. Road, Taipei, Taiwan 106 \\ jyjun@ntut.edu.tw
}

Nanotechnology, which can be defined as the understanding and control of matter at dimensions between approximately 1 and $100 \mathrm{~nm}$, has attracted enormous research and public interest for just about two decades. Nanotechnology is not a single process; neither does it involve a specific type of material. Instead, the term covers all aspects of the production of materials, devices, and systems by manipulating matter at the nanoscale. Encompassing nanoscale science, engineering, and technology, nanotechnology involves imaging, measuring, modeling, and manipulating matter at this length scale. Nanostructured thin films display unique phenomena, thus enabling the improvement of traditional applications or the development of novel applications. ${ }^{1}$ The fabrication, characterization, modeling, and manipulation of nanostructured thin films are essential to further scientific progress.

The Nanostructured Thin Films III: Fabrication, Characterization, and Application Conference was held on August 4 and 5, 2010 in San Diego, California. Up to 39 papers were submitted encompassing topics covering many relevant aspects of nanostructured thin films, from modeling, fabrication, and characterization to practical applications. As such, 10 sessions were arranged, namely Columnar Morphology I, II and III, Sensor Applications, Theoretical Optics, Plasmonics, Fabrication and Characterization I and II, and Applications I and II.

This special section comprises a set of selected papers which have been expanded from those presented during the Nanostructured Thin Films III: Fabrication, Characterization, and Application Conference.

The guest editors do thank Professor Akhlesh Lakhtakia for co-chairing the conference, all the authors for their contribution to this special section and those that participated in the Nanostructured Thin films III: Fabrication, Characterization, and Application Conference and contributed to its valuable proceedings.

\section{Reference}

1. R. J. Martín-Palma and A. Lakhtakia, Nanotechnology: A Crash Course, SPIE Press, Bellingham, WA (2010). 